\title{
NEUROCIÊNCIA E CIÊNCIAS SOCIAIS: UMA REVISÃO DOS CONCEITOS DO NEUROMARKETING, DA NEUROERGONOMIA E DO NEURODESIGN
}

\author{
Melissa Marin Vasquez \\ UNESP - FAAC \\ melissamava@gmail.com \\ Liara Mucio De Mattos \\ UNESP - FAAC \\ liaramattos@gmail.com \\ Guilherme Da Silva Bertolaccini \\ UNESP - FAAC \\ guilhermebertolaccini@gmail.com
}

\author{
Paula Da Cruz Landim \\ UNESP - FAAC \\ Luis Carlos Paschoarelli \\ UNESP - FAAC \\ paschoarelli@faac.unesp.br \\ Fausto Orsi Medola \\ UNESP - FAAC \\ fausto.medola@faac.unesp.b
}

sustenta na ciência e nos conhecimentos adquiridos com os avanços tecnológicos, visando o desenvolvimento de novos produtos e uma melhor compreensão de seus usuários. Uma das importantes áreas que o Design se aliou é a neurociência. Esta foi capaz de revelar novas possibilidades para conhecer e compreender melhor a natureza da cognição e da conduta humana. Deste modo, a neurociência proporciona uma alternativa cientifica para explicar o comportamento das pessoas durante o uso de produtos e sistemas. Este estudo tem como objetivo compreender a aplicação da neurociência nas disciplinas de marketing, ergonomia e design, além de estabelecer uma reflexão introdutória a respeito da incorporação da neurociência no design nas esferas acadêmica e profissional, além de estabelecer uma reflexão introdutória a respeito da incorporação da neurociência no design nas esferas acadêmica e profissional.

Palavras-chave: Ergonomia, Cognição, Neuromarketing, Neuroergonomia, Neurodesign.

Abstract: The design is in of constantly evolution, increasingly supporting by science and the knowledge gained with technological advances in the development of new products and a better understanding of its members. One of the important areas that $q$ Design is allied neuroscience. This was able to reveal new possibilities to know and better understand the nature of cognition and human behavior. Thus is providing a scientific approach to the person and better understand the behavior, conditions and user interests against many situations, spaces or products. This study aims to understand the application of neuroscience in marketing disciplines, 
ergonomics and design, as well as establishing an introductory reflection on the neuroscience of incorporation into the design in academic and professional spheres, through a literature review and that will serve as a basis for understanding of the applicability of neuroscience in the development of design projects.

Keywords: Ergonomics, Cognition, Neuromarketing, Neuroergonomia and Neurodesign

\section{INTRODUÇÃO}

Nos últimos anos, a ciência e a tecnologia têm influenciado o estilo de vida da sociedade, sendo a tecnologia o aspecto que tem modificado mais substancialmente as atividades humanas, tais como comunicação, mobilidade, saúde, entre outros. Segundo Acevedo (1998), a tecnologia é uma das características mais relevantes dos tempos atuais e é inquestionável sua importância em todos os âmbitos sociais. $O$ autor considera a tecnologia como um fator essencial no logro de metas de caráter social, cultural, econômico e político. A ciência e a tecnologia se relacionam mutuamente, essa conexão de conhecimentos tanto teóricos como práticos se aplica na interpretação e transformação do entorno e solução de problemas (ACEVEDO, 1998).

No que se refere às disciplinas do design, os avanços tecnológicos e científicos têm propiciado a incorporação de novas ferramentas e saberes que contribuem para a geração de novos cenários de ação (HERRERA, 2012).

Para Herrera (2012) os avanços tecnológicos e científicos têm modificado os meios e as ferramentas na prática do design, transformando alguns planejamentos teóricos e metodológicos da disciplina. Dessa forma, o design se encontra em evolução constante, cada vez mais sustentado na ciência e nos conhecimentos dos avanços tecnológicos para o desenvolvimento de novos produtos.

$\mathrm{Na}$ busca pela compreensão do comportamento, condições e interesses do usuário frente a diversas situações, espaços ou produtos, Herrera (2012) afirma que algumas disciplinas das ciências sociais têm se sustentado na neurociência, uma vez que esta abre novas possibilidades de compreensão da natureza da cognição e da conduta humana, brindando com uma aproximação cientifica à pessoa. Segundo o autor, cabe aos pesquisadores e profissionais das diferentes disciplinas da área das ciências sociais - design, economia, entre outros - incorporar os avanços neurocientíficos na prática.

O presente estudo tem como objetivo analisar e compreender a aplicação da neurociência em algumas disciplinas - marketing, ergonomia e design, bem como estabelecer uma reflexão preliminar sobre a incorporação da neurociência no design nos âmbitos acadêmico e profissional por meio de uma revisão teórica pautada na compreensão da aplicabilidade da neurociência no desenvolvimento de projetos de produtos e sistemas 


\section{GENERALIDADES DA NEUROCIÊNCIA}

As neurociências são universos de conhecimentos sobre o sistema nervoso e estabelecem um campo de confluência para as diversas perspectivas que se têm aproveitado no seu estudo (CORSI e CABRERA, 2004). Segundo Corsi e Cabrera (2004), o objeto de estudo das neurociências é o sistema nervoso que é o coordenador de todas as funções do organismo desde as funções automáticas até as funções mais complexas (como o pensamento, a linguagem e o comportamento). Esses autores afirmam que através do processamento de toda informação que vem do exterior ambiente, outros seres vivos, etc-, o sistema nervoso toma decisões e executa ações que por sua vez afetam seu entorno.

Para Jessel et al. (1997), o objetivo central das neurociências é tentar explicar como atuam milhões de células nervosas individuais no encéfalo para produzir o comportamento e como estas células são influenciadas pelo meio ambiente e outros indivíduos. Segundo Gimenez e Murillo (2007) as neurociências têm se diferenciado pelo enfoque sintético e integrador das ciências que se dedicam ao estudo do sistema nervoso, o qual integra estímulos internos e externos à pessoa e, através da experiência cognitiva, emocional e motivação acumulada, dá lugar a uma resposta dentro e fora do organismo que pode ser influenciada por processos psíquicos de caráter cognitivo ou afetivo, de acordo com a experiência do indivíduo.

Correa (2008) define a neurociência como uma disciplina que faz uso do conhecimento da estrutura e funcionamento do cérebro para a solução de problemas práticos. $\mathrm{O}$ autor enfatiza os diferentes conhecimentos que nutrem a neurociência tais como: psicologia clínica, reabilitação neuropsicológica e a ergonomia, destacando seu forte caráter multidisciplinar, o que facilita o surgimento de novos campos de aplicação dos conhecimentos do cérebro a outros ramos relacionados com tomada de decisões, aprendizagem, trabalho, entre outros.

Squire et al. (2012) descreve a neurociência como uma coleção de ciências multidisciplinares que analisam o sistema nervoso para entender a base biológica do comportamento. Os autores afirmam que hoje a neurociência se expande para um amplo ramo de pesquisa que vai desde a biologia molecular das células nervosas até a base biológica do comportamento, emoção e cognição, e suas alterações.

Os estudos correspondentes à área das neurociências vêm crescendo de maneira rápida, em grande parte devido à pluralidade de disciplinas. Esta pluralidade tem estimulado a origem de diversos enfoques, sendo um exemplo a disciplina da psicologia que, através dos conhecimentos da biologia e da neurociência, tem definido tópicos de estudo - neurociência cognitiva- que são aplicáveis ao desenvolvimento de tecnologia, tais como o uso de computadores (SQUIRE, et al. 2012. p. 10). Do mesmo modo, da neurociência cognitiva surgem outros enfoques tais como Neuromarketing, a Neuroergonomía e o Neurodesign, os quais serão expostos a seguir.

\subsection{Neurociência Cognitiva}

A neurociência cognitiva é descrita por Escera (2004) como a disciplina que busca entender a função cerebral em suas atividades mentais: percepção; memória e 
linguagem. O termo foi utilizado pela primeira vez em um curso desenvolvido pelos cientistas Gazzaniga e Miller no instituto Cornell Medical College, em 1976, sobre as bases biológicas da cognição humana, as quais eram consideradas uma aproximação pertinente para estudar sujeitos com técnicas das ciências do cérebro de acordo com os métodos das ciências cognitivas (Escera, 2004. p. 2). Segundo Escera (2004) a neurociência cognitiva não é muito diferente das neurociências entendidas do modo mais amplo. Fuster (2000) afirma que toda neurociência é cognitiva, enquanto Escera (2004) declara que a "neurociência cognitiva constitui uma forma de entender as relações cérebro-cognição com a identidade pragmática própria, diferenciada da neurociência em geral e das disciplinas psicológicas e psicobiológicas em particular[...] e seu antecedente mais próximo foi a Psicologia Fisiológica que se havia estabelecido como uma disciplina consolidada".

No transcurso do tempo temos visto em alguns estudos (PEREIRA \& LAGO, 2015; GIRAUDET, ET AL., 2015; ALEXIOU, ET AL.,2009) que a neurociência cognitiva tem aumentado os campos de atuação como a atenção, a visão, a memória, entre outras. Segundo Escera (2004) são quatro os âmbitos onde a neurociência cognitiva está avançando. Atualmente o mundo tecnológico está evoluindo constantemente, portanto uma das primeiras necessidades é a de combinar métodos de alta precisão espacial (ressonância magnética funcional) que permitem visualizar as zonas cerebrais que se ativam enquanto a pessoa está realizando alguma tarefa cognitiva (VENDRELL, ET AL., 1995); e maior resolução temporal (magnetoencefalografía), que permite registrar os campos magnéticos gerados pelo fluxo de uma corrente elétrica que permite explorar o funcionamento cerebral (CAPILLA, ET AL., 2004). Posteriormente, a área da psiquiatria e os estudos sobre transtornos de desenvolvimento e alterações da percepção são outros dois âmbitos nos quais a neurociência cognitiva está se desenvolvendo atualmente.

Finalmente, para Escera (2004. p. 14) o outro âmbito ao qual se refere à chamada neurociência cognitivo-social, campo de pesquisa que visa busca entender os fenômenos comportamentais em três perspectivas: social, a partir dos fatores motivacionais e sociais que influenciam o comportamento e a experiência; o cognitivo, que se interessa pelos mecanismos de processamento de informação; e o grau neurológico, que aborda os mecanismos cerebrais relacionados ao processo cognitivo.

Maureira (2010) afirma que a neurociência permite entender os conhecimentos da economia, educação e a sociologia a partir dos novos pontos de vista. Esses pontos de vista têm dado lugar a diferentes áreas do conhecimento que associam diversas disciplinas como, por exemplo, o Neuromarketing, a Neuroergonomía e o Neurodesign.

\subsection{Neuromarketing}

A economia foi uma das primeiras áreas das ciências sociais a introduzir a metodologia da neurociência em suas pesquisas, o que permitiu entender o comportamento do consumidor e os conceitos relacionados à tomada de decisões, confiança e memória (LEE, et al., 2007). Da integração entre a economia e os conhecimentos da neurociência surge o neuromarketing, disciplina considerada por Lee, et al. (2007) como uma subárea da economia. O neuromarketing tem como 
objetivo estudar o comportamento humano nos mercados, através da informação clínica e os mecanismos cerebrais (FUGATE, 2007). Para o neuromarketing, o equilíbrio da comercialização está mudando a favor do consumidor, o acesso às ferramentas de registro (ou análise) de sinais nervosos permite fazer um analise do que verdadeiramente quer o consumidor. Segundo Walton (2004), já se encerraram os estudos de mercado nos quais as pessoas diziam o que as empresas "queriam escutar", o que evita o problema de depender dos auto-relatos. Além disso, é difícil entender as razões preferenciais dos consumidores e suas decisões de consumo (FUGATE, 2007. p. 386). O potencial do neuromarketing é reduzir os fracassos do marketing através de imagens cerebrais do consumidor que permitam conhecer como atender expectativas e ativar sensações positivas.

Dentre os métodos de análise do neuromarketing se encontram as técnicas como a eletromiografia, a magneto encefalograma e tomografia que geram imagens neurológicas que permitem evidenciar o processamento da informação relacionado às decisões de compra. Convencionalmente, são utilizadas estratégias como o focus group e entrevistas para investigação de satisfação do consumidor. Fugate (2007. p. 387) afirma que os sujeitos podem utilizar um dispositivo de imagem cerebral enquanto estão assistindo imagens ou videoclipes de uma nova campanha promocional. Segundo o autor, dependendo de qual área cerebral é ativada, podem ser reconhecidos padrões de pensamento do sujeito. Através do uso destas ferramentas de imagens neurais e dos conhecimentos da neurociência cognitiva, os anúncios publicitários podem ser adaptados com o objetivo de estimular as regiões cerebrais responsáveis pelas emoções -paixão, entusiasmo, alegria- que a agência de marketing está buscando. Assim, os profissionais podem adequá-los de forma que afinem as mensagens dos anúncios para estimular a compra (MCCONNON; STEAD, 2007).

\subsection{Neuroergonomia}

A neuroergonomia é definida como o estudo do cérebro humano e sua relação com o desempenho no trabalho e nas atividades cotidianas. Através da integração de teorias e princípios da ergonomia e da neurociência, provê informação valiosa sobre a função cerebral e comportamentos em entornos naturais (PARASURAMAN, 2003). Segundo Correa (2008), o psicólogo israelita Daniel Kahneman (1973) estabeleceu as bases da neuroergonomia através dos estudos sobre a dilatação da pupila como índice de sobrecarga mental enquanto o sujeito realiza uma tarefa cognitiva complexa. De acordo com Correa (2008), a neuroergonomia utiliza os conhecimentos sobre o cérebro para a melhora da interação do sistema de trabalho, além de predizer o estado físico do trabalhador (sudorese, frequência cardíaca e respiratória, atividade muscular e cerebral) e estados cognitivos (fatiga mental, sonolência, entre outras).

A neuroergonomia estuda a estrutura e a função do cérebro além das tarefas básicas usadas pela psicologia cognitiva e a neurociência. Uma melhor compressão do cérebro pode proporcionar diretrizes para o fortalecimento da apresentação da informação e design de tarefas, otimização de alertas e sinais, entre outras (PARASURAMAN \& WILSON, 2008). Os estudos de neuroergonomia baseiam-se em técnicas de neuroimagens que permitem entender a estrutura, os mecanismos e as 
funções cerebrais durante o trabalho (MEHTA \& PARASURAMAN, 2013). As técnicas aplicáveis na neuroergonomia são divididas em duas categorias: O EEG (eletroencefalograma), que indica a atividade cerebral; e a técnica de EPRs (EventRelated Potentials), que provê uma medida objetiva para o seguimento de progressão ou respostas a terapias (TOMSAK, 2005. p 226-227). O EEG representa a atividade elétrica dos neurônios que respondem aos estímulos cognitivos mostrando uma excelente resolução temporal em milissegundos das mudanças cerebrais eletromagnéticas (MEHTA \& PARASURAMAN, 2013. p. 1).

Uma área importante dentro da neuroergonomia é a realidade virtual, que consiste em gerar diferentes cenários virtuais com múltiplas aplicações científicas, como por exemplo o comportamento de um trabalhador em situações de perigo ou facilitar o treinamento em tarefas complexas (CORREA, 2008). Em síntese, a ergonomia evoluiu de uma disciplina que visa melhorar a eficiência no trabalho a uma que objetiva melhorar o bem-estar das pessoas. A aplicação da neurociência cognitiva permite entender como as pessoas interagem com os diferentes sistemas de trabalho, abordando bases no conhecimento neural relacionadas com o rendimento físico e cognitivo que as avaliações ergonômicas clássicas não podem captar (MEHTA \& PARASURAMAN, 2013. p. 8).

\subsection{Neurodesign}

A $O$ design é uma atividade projetiva que tem como objetivo responder às necessidades, limitações e interesses do usuário por meio do desenvolvimento de produtos, espaços e serviços. Na maioria dos projetos de design se parte do enfoque, Design centrado no usuário, que visa melhorar a condição social e individual do ser humano através da incorporação dos avanços da ciência e a tecnologia (HERRERA, 2012. p. 5).

Nos últimos anos se tem experimentado diversas mudanças (sociais, tecnológicas, culturais, entre outras) as quais tem orientado a teoria do design. Esta disciplina se encontra em estado de evolução constante, junto com as exigências dos usuários, o que tem permitido a geração de mudanças e o surgimento de novas teorias. Uma das novas teorias e práticas do design é o uso dos conhecimentos e técnicas da neurociência para o desenvolvimento de produtos (GUTIERREZ, et al., 2011).

Para Gutiérrez, et al. (2011) a neurociência no design requer um esforço para integrar conhecimentos no processo de design de forma a favorecer o desenvolvimento de produtos mais sustentáveis nos aspectos psicológicos, sociais e culturais. Para os autores, o neurodesign articula o conhecimento da neurociência cognitiva e do design para a criação de produtos que satisfaçam as necessidades do usuário, adotando uma postura que integre o cérebro, o comportamento e a cultura que constituem o indivíduo e o processo de uso de um produto em um dado contexto.

Herrera (2012), propõe três campos de ação para o neurodesign, tendo em conta que o objeto final do design é o usuário e que a experiência determina pelos processos neurais dele: a aplicação de conhecimentos de neurociência que são úteis para melhorar a prática do design; a utilização de ferramentas de pesquisa em 
neurociência de forma a contribuir para o desenvolvimento e avalição de produtos de design; e o desenvolvimento de novos produtos a partir de dispositivos desenvolvidos com teorias fundamentadas na neurociência.

As ferramentas utilizadas para a pesquisa do neurodesign são baseadas em métodos não invasivos tais como a tomografia computorizada, a ressonância magnética funcional e a eletroencefalografia, as quais permitem ter informação objetiva com respeito à experiência do usuário na interação do design. Portanto, a neurociência abre novas possibilidades para o estudo e conhecimento do comportamento, emoções e cognição dos usuários e suas interações com produtos, ambientes e sistemas no processo de uso.

\section{DISCUSSÕES E CONSIDERAÇÕES FINAIS}

A neurociência e seus recentes avanços contribuem de forma importante para as áreas das ciências sociais que buscam alternativas para melhorar as exigências humanas. É importante destacar que a evolução tecnológica tem possibilitado o uso de ferramentas que são capazes de estudar e registrar o que acontece no cérebro humano em diferentes contextos. Estas ferramentas nos permitem verificar mudanças na atividade cognitiva tais como a atenção, estados de alerta, emoções, entre outros, que podem ser uteis no estudo e avaliação de campanhas publicitárias, postos de trabalho ou interfaces gráficas (HERRERA, 2012).

Há um constante interesse em conhecer os processos mentais que envolvem a percepção, cognição e as experiências das pessoas em diferentes situações, o que tem permitido que diferentes disciplinas das ciências sociais adotem conhecimento das neurociências na tentativa de melhor entender o comportamento humano. As disciplinas aqui expostas -marketing, ergonomia e design- tem incorporado os conhecimentos da neurociência para analisar as atitudes dos indivíduos frente a algumas situações e no entanto as finalidades de cada uma se diferenciam. Uma síntese das três abordagens e suas relações com o Design é apresentada na Tabela 1.

Embora os três conceitos utilizem as mesmas ferramentas para a análise do indivíduo, eles possuem objetivos, aplicações e analises diferentes. Em primeiro lugar o marketing analisa o sujeito como um consumidor e, desta forma, o neuromarketing utiliza os conhecimentos das neurociências para compreender o comportamento, orientar ou adotar uma conduta especifica na decisão de compra (GLIMCHER \& RUSTICHINI, 2004). Por sua vez, a Neuroergonomia e o Neurodesign buscam melhorar o bem-estar das pessoas, não obstante os dois buscam entender o indivíduo de jeito diferentes. A neuroergonomia visa examinar e estudar como o cérebro se comporta durante a realização de uma atividade na vida cotidiana e em diferentes ambientes, estudando aspectos como tempo de atenção, carga mental, processo motor entre outros (PARASURAMAN \& RIZZO, 2006). Já o neurodesign busca entender o indivíduo como um usuário que percebe diferentes estímulos quando interage com um produto e as experiências que geram, e assim, utilizar os resultados dessas análises no design formal dos produtos (GUTIERREZ, et al., 2011). 
Quadro 1 - Comparação entre Neuromarketing, Neuroergonomia e Neurodesign

\begin{tabular}{|c|c|c|c|}
\hline & NEUROMARKETING & NEUROERGONOMIA & NEURODESIGN \\
\hline Pessoa & Consumidor & Trabalhador & Usuário \\
\hline Objetivo & $\begin{array}{l}\text { Melhora de estratégias } \\
\text { de marketing e promoção } \\
\text { de vendas }\end{array}$ & $\begin{array}{l}\text { Examinar e estudar como } \\
\text { o cérebro realiza uma } \\
\text { tarefa na sua vida } \\
\text { cotidiana e em diferentes } \\
\text { ambientes. }\end{array}$ & $\begin{array}{l}\text { Entender como o usuário } \\
\text { percebe os diferentes } \\
\text { estímulos e cria as } \\
\text { experiências que tem } \\
\text { quando entra em contato } \\
\text { com os objetos. }\end{array}$ \\
\hline Aplicações & $\begin{array}{l}\text { Testar a efetividade de } \\
\text { uma publicidade. } \\
\text { Seleção de logos/marcas. } \\
\text { Seleção de tipos de } \\
\text { médios de divulgação. }\end{array}$ & $\begin{array}{l}\text { Avaliação de processos } \\
\text { de automatização, } \\
\text { engenheira neural para } \\
\text { pessoas com deficiência, } \\
\text { desenvolvimento de } \\
\text { métodos de seleção e } \\
\text { formação de novos } \\
\text { postos de trabalho. }\end{array}$ & $\begin{array}{l}\text { Desenvolvimento de } \\
\text { novos produtos. } \\
\text { Desenvolvimento de } \\
\text { interfaces gráficas. }\end{array}$ \\
\hline $\begin{array}{l}\text { Ferramentas } \\
\text { utilizadas }\end{array}$ & $\begin{array}{l}\text { Tomografia } \\
\text { computorizada; } \\
\text { Ressonância magnética } \\
\text { funcional; } \\
\text { Eletroencefalografia }\end{array}$ & $\begin{array}{l}\text { Tomografia } \\
\text { computorizada; } \\
\text { Ressonância magnética } \\
\text { funcional; } \\
\text { Eletroencefalografia }\end{array}$ & $\begin{array}{l}\text { Tomografia } \\
\text { computorizada; } \\
\text { Ressonância magnética } \\
\text { funcional; } \\
\text { Eletroencefalografia }\end{array}$ \\
\hline
\end{tabular}

Fonte: Elaborado pelos autores, com base na pesquisa realizada

Considerando a neurociência aplicada às ciências sociais aqui expostas, é importante perguntar se cada nova proposta conceitual é necessária. Segundo Pasaraman (2003. p. 469), os leitores têm direito a ser céticos sempre que leem "neuro" como prefixo dado que pode desaparecer no futuro. O autor considera que, futuramente, os pesquisadores considerarão os conhecimentos da neurociência sem referir-se necessariamente a neuromarketing, neuroergonomia ou neurodesign. No entanto, é importante considerar os conhecimentos das neurociências nestas disciplinas como uma nova forma de estudar e compreender os diferentes fatores físicos e cognitivos do ser humano em função de suas diversas necessidades, bem como o mercado e as mudanças sociais que acontecem no decorrer do tempo.

Por fim, destaca-se que são poucos os estudos que incluem as bases teóricas da neurociência, nesse sentido, pesquisadores e profissionais das áreas das ciências sociais - em especial - devem buscar vincular os avanços tecnológicos e conhecimentos científicos nas aplicações práticas. 


\section{REFERÊNCIAS}

ACEVEDO, Germán Rodríguez. Ciencia, Tecnología y Sociedad: una mirada desde la Educación en Tecnología. Revista Iberoamericana de Educación, 1998, n. 18, p. 107 144.

ALEXIOU, Katerina, et al. Exploring the neurological basis of design cognition using brain imaging: some preliminary results. Design Studies, 2009, v. 30, n. 6, p. 623647.

CAPILLA-GONZÁLEZ, A., et al. La magnetoencefalografía en los trastornos cognitivos del lóbulo frontal. Revista de neurología, 2004, v. 39, n. 2, p. 183-188.

CORREA, A. Neurociencia aplicada: el cerebro al servicio de la humanidad. Departamento de Psicología Experimental y Fisiología del Comportamiento Universidad de Granada, 2008.

CORSI, Maria; CABRERA, María Corsi. Aproximaciones de las neurociencias a la conducta. UNAM, 2004.

DAROFF, Robert B.; AMINOFF, Michael J. Encyclopedia of the neurological sciences. Academic press, 2014.

DE LA BARRERA, María Laura; DONOLO, Danilo. Neurociencias y su importancia en contextos de aprendizaje. Revista digital universitaria, 2009, v. 10, n. 4, p. 1-10.

ESCERA, Carles. Aproximación histórica y conceptual a la Neurociencia Cognitiva.

Cognitiva, 2004, v. 16, n. 2, p. 141-61.

FUSTER, Joaquin M. The Module: Crisis of a Paradigm-The New Cognitive Neurosciences, Michael S. Gazzaniga, Editor-in-Chief Cambridge, Massachusetts: MIT Press (1999). Neuron, 2000, v. 1, n. 26, p. 51-53.

FUGATE, Douglas L. Neuromarketing: a layman's look at neuroscience and its potential application to marketing practice. Journal of Consumer Marketing, 2007, v. 24, n. 7, p. 385-394.

GIMENEZ-AMAYA, José Manuel; MURILLO, José Ignacio. Mente y cerebro en la Neurociencia contemporánea. Una aproximación a su estudio interdisciplinar. 2007.

GIRAUDET, Louise, et al. The neuroergonomic evaluation of human machine interface design in air traffic control using behavioral and EGG/ERP measures. Behavioural brain research, 2015, 294: 246-253.

GLIMCHER, Paul W.; RUSTICHINI, Aldo. Neuroeconomics: the consilience of brain and decision. Science, 2004, v. 306, n 5695, p. 447-452.

GUTIERREZ, María Jesús Ávila, et al. Neurodiseño y neurousabilidad: un nuevo enfoque del diseño desde la perspectiva de la Neurociencia. Sevilla Técnica, 2011, n 37, p. 4245 .

HERRERA BATISTA, Miguel Ángel. El neurodiseño como una nueva práctica hacia el diseño científico. No Solo Usabilidad, 2012, n 11.

KANDEL, Eric R. Jessell, et al. Neurociencia y conducta. 1997.

KAHNEMAN, Daniel. Attention and effort. Englewood Cliffs, NJ: Prentice-Hall, 1973. 
LEE, Nick; CHAMBERLAIN, Laura. Neuroimaging and psychophysiological measurement in organizational research. Annals of the New York Academy of Sciences, 2007, v. 1118 , n 1, p. 18-42.

LEE, Nick; BRODERICK, Amanda J.; CHAMBERLAIN, Laura. What is 'neuromarketing'? A discussion and agenda for future research. International Journal of Psychophysiology, 2007, vol. 63, no 2, p. 199-204.

MAUREIRA, Fernando. La neurociencia cognitiva: Una ciencia base para la Psicología. Psiquiatría Universitaria, 2010, v. 6, n 4, p. 449-453.

MCCONNON, A.; STEAD, D. If I only had a brain scan. Business Week, 2007, v. 4018, n 19, p. 22.

MEHTA, Ranjana K.; PARASURAMAN, Raja. Neuroergonomics: a review of applications to physical and cognitive work. Frontiers in human neuroscience, 2013, v. 7, p. 889.

PARASURAMAN, Raja. Neuroergonomics: Research and practice. Theoretical Issues in Ergonomics Science, 2003, v. 4, n 1-2, p. 5-20.

PARASURAMAN, Raja; RIZZO, Matthew (ed.). Neuroergonomics: The brain at work. Oxford University Press, 2006.

PARASURAMAN, Raja; WILSON, Glenn F. Putting the brain to work: Neuroergonomics past, present, and future. Human Factors: The Journal of the Human Factors and Ergonomics Society, 2008, v. 50, n. 3, p. 468-474.

PEREIRA, Verónica Crespo; LAGO, Beatriz Legerén. Neurogaming: El papel de la neurociencia en la industria del videojuego, 2015

SQUIRE, Larry, et al. (ed.). Fundamental neuroscience. Academic Press, 2012.

TOMSAK, Robert L. Encyclopedia of the Neurological Sciences. 2005.

VENDRELL, Pere; JUNQUÉ, Carme; PUJOL, Jesús. La resonancia magnetica funcional: Una nueva tecnica para el estudio de las bases cerebrales de los procesos cognitivos. Psicothema, 1995, v. 7, n. 1, p. 51-60.

WALTON, C. The brave new world of neuromarketing is here. B\&T (Australia), 2004, v. 19, p. 22. 